\title{
Multiband Monopole Antenna with Sector-Nested Fractal
}

\author{
Jie Zhang, Wei Zhang \\ Joint-Lab. of JNU-IREE RAS, College of Information Science and Technology, Ji Nan University (JNU), Guangzhou, \\ China \\ Email: 1079874683@qq.com
}

Received 17 June 2015; accepted 5 July 2015; published 8 July 2015

Copyright @ 2015 by authors and Scientific Research Publishing Inc.

This work is licensed under the Creative Commons Attribution International License (CC BY).

http://creativecommons.org/licenses/by/4.0/

(c) (i) Open Access

\section{Abstract}

A kind of novel multi-frequency monopole antenna with sector-nested fractal is proposed and designed, which is nested with a series of similar circular sector elements. By means of the trapeziform ground plane with the tapered CPW (coplanar waveguide) feeder in the middle, the antenna's radiation performance is greatly improved. The antennas can synchronously operate in three frequencies, covering the working frequency bands of WLAN/WiMAX, 2.44 GHz/3.5 GHz/5.2 GHz $5.8 \mathrm{GHz}$. The pattern and impedance measurements of antenna show a good performance over the WLAN/WiMAX band; it possesses a near omni-directional characteristic and good radiation efficiency. Moreover, the antenna is miniature and its design idea can be easily applied into other types of nested structure, the features of which make the proposed antenna have a promising application in other fields.

\section{Keywords}

Sector-Nested, Fractal Antenna, Trapeziform Ground Plane, Multi-Frequency

\section{Introduction}

Development of high data rates wireless communication system and the increase of the communication frequency bands put forward the demand for multi-band antennas. In a fixed space, there usually exist many different communication systems, such as wireless local area network system, mobile communication system, etc. These wireless systems need the antennas which could work on different frequencies and modes. For example the working frequencies for the wireless local area network (WLAN) are $2.4 \mathrm{GHz}$ and $5.2 \mathrm{GHz}$, and those for the GSM wireless mobile terminal are $900 \mathrm{MHz}$ and $1800 \mathrm{MHz}$. So we really need an antenna, it can meet the requirements of different applications for the band and also can guarantee the gain of antenna. Specifically, an 
antenna can work on different frequency bands, and the corresponding radiation characteristics within each frequency band can be guaranteed.

There are many methods proposed to realize the miniaturization characteristic of the antennas, including resonant units [1] [2], different patterns [2] [3], antenna loading [4], surface of the slot [5], electronic reconfigurable [6], and adopting reconstructed structure [7]. These methods are so specialized that only some aspects of the demand can be met, while the overall performance of the antenna is decreased in order to satisfy the communication system. Recently, people pay more and more attention on fractal technique, which is a new method to achieve multiband.

Fractals have self-similar shapes and can be subdivided in parts such that each part is a reduced size copy of the whole. The self-similarity of fractals is the cause of multi-band property and their complicated shapes contribute to the design of antennas with smaller size. Fractals have convoluted and jagged shapes, and these discontinuities will increase bandwidth and weaken the effective radiation of antennas. The space-filling property of fractal makes curves which have long electrical length be fitted into a compact physical volume. The typical fractal antennas in recent years include Sierpinski fractal antenna [8], Koch fractal antenna [9] and Minkowski antenna [10].

In this paper, a novel sector-nested fractal antenna is proposed, which is a single-plane printed monopole antenna fed by the trapeziform ground plane with CPW. The antenna can synchronously operate in three frequencies, covering the working frequency bands of WLAN/WiMAX $(2.44 \mathrm{GHz} / 3.5 \mathrm{GHz} / 5.2 \mathrm{GHz}-5.8 \mathrm{GHz})$. The paper is organized as follows. Section 2 describes the proposed design method and short formulation. Section 3 presents results of simulations using Ansoft HFSS. Section 4 concludes the findings of the paper.

\section{Formulation and Antenna Design}

\subsection{Formulation}

In recent several years, fractal has caused a fast-growing interest of scientists. These structures (systems) description cannot be presented using traditional derivative equations of integer order. More exactly these processes and objects are quantitatively described by integration-differentiation operators of fractional order $D \alpha[f(t)]$, where $-1<\alpha<1$. Presence the fractional derivative in equations is decided to interpret as reflection of specific peculiarity of processes system which is memory or non-Markovian attribute.

The fractal and processes into them can be described by operators with real degree [11]. Diffusion here is anomalous diffusion and it is described by fractional diffusion equation.

$$
\frac{\partial W}{\partial t}={ }_{0} D_{t}^{1-\alpha} K_{\alpha} \frac{\partial^{2}}{\partial x^{2}} W(x, t)
$$

where $W(x, t)$ is a function of probability density depended in general on interaction geometry peculiarities, $K_{\alpha}$ is generalized diffusion coefficient and ${ }_{0} D_{t}^{1-\alpha}=\frac{\partial}{\partial t}{ }_{0} D_{t}^{-\alpha}$ is operator of Riemann-Liouville which is determined by the following integral relationship $(0<\alpha<1)$ [12]. It is direct continuation of Cauchy multiple integral for arbitrary complex $\alpha$ with $\operatorname{Re}(\alpha)>0$.

$$
{ }_{0} D_{t}^{1-\alpha} W(x, t)=\frac{1}{\Gamma(\alpha)} \frac{\partial}{\partial t} \int_{0}^{t} \mathrm{~d} t^{\prime} \frac{W\left(x, t^{\prime}\right)}{\left(t-t^{\prime}\right)^{1-\alpha}}
$$

Therefore integration-differentiation nature of fractional operator of Riemann-Liouville ${ }_{0} D_{t}^{1-\alpha}$ accordingly to (2) and with integral kernel of form $M(t) t^{\alpha-1}$ is providing non-Markovian nature of sub-diffusion process defined by diffusion fractional Equation (1).

\subsection{Antenna Structure}

The sector-nested fractal antenna is evolved from multiple-ring fractal antenna [13] and the evolution process is presented in Figure 1. Antenna radiator is nested by multiple sector loops and the novel sector-nested antenna can be obtained. The two antennas in Figure 1 can be considered that the sector or circle metal-patches are caverned out and orderly nested from larger to smaller. 

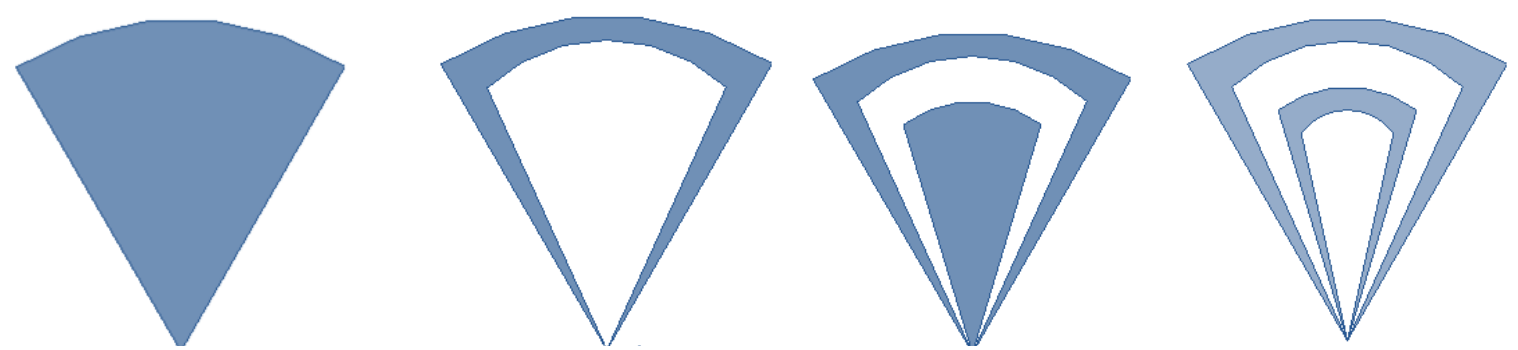

Figure 1. The evolution process of sector-nested fractal antenna.

The novel sector-nested fractal antenna is shown in Figure 2, the substrate material is adopted FR-4 with relative permittivity $\varepsilon_{r}=4.4 \mathrm{~mm}$ and $1.5 \mathrm{~mm}$ thickness. The dark part is metal and the white part is the substrate. The number of nested fan is set to $n=4$, and at the same time the segment heights are $h_{1}, h_{2}, h_{3}, h_{4}$, the vertex angles for the antenna are $\theta_{1}, \theta_{2}, \theta_{3}, \theta_{4}$, and metal thickness is $2 \mathrm{~mm}$. As we can see, the antenna has two main parts-metal radiator and trapeziform ground plane. Here the trapeziform ground plane has three functions: the first is acting as a ground plane for the monopole and the CPW, the second is acting as a radiating element, and the third is acting as a component to form the distributed matching network with the monopole together.

\section{Antenna Simulation}

According to previous research, fractal fan-nested antenna with the fan unit in different sizes will have an effect on the reflection loss of multiband, and the mainly factors that influence the pass-band center frequency and bandwidth are the height of the nested fan and vertex angles. Below we will change the height and angle of the sector, and analyze how these two variables affect the performance of the antenna.

\subsection{The Height of the Nested Fan}

Set the fan-nested fractal parameters as follows: $h_{2}=17 \mathrm{~mm}, h_{3}=14 \mathrm{~mm}, h_{4}=12 \mathrm{~mm}, \theta_{1}=80^{\circ}, \theta_{2}=70^{\circ}$, $\theta_{3}=60^{\circ}, \theta_{4}=50^{\circ}$. The first investigated parameter for the structure is $h_{1}$, its effects on the antenna's $\mathrm{S}_{11}$ and bandwidth in $1 \mathrm{~mm}$ steps are shown in Figure 3. Because the outer fan is in the largest size, so it plays a decisive role on the low band of the center frequency, bandwidth and reflection loss.

The experiential formula of deciding antenna size can be established by simulation, which indicates the relation between the outer fan side-length $\left(h_{1}\right)$ and the first resonance frequency band $\left(f_{1}\right)$, as shown in the following:

$$
f_{1}=0.11 c / h_{1}
$$

In formula (3), the speed of light $c=3 \times 10^{8} \mathrm{~m} / \mathrm{s}$. Thus, the outer most fan height and first resonance frequency has an inversely proportional relationship, that is, with the increase of $h_{1}$, the corresponding reflection loss of the first band center frequency will be decreased.

Keep the outer fan height and other variables constant and respectively change the second and the third height of the nested fan, the results of which are shown in Figure 4 and Figure 5, and the relevant data statisticsis shown in Table 1. The electromagnetic analysis of the antenna is done by means of finite-difference time-domain (FDTD) using the commercially available HFSS.

Table 1 demonstrates that: 1 ) With the increase of $h_{1}$ and $h_{2}$ respectively, the center frequency of the low pass-band is shifting obviously, and two dimensions $h_{1}$ and $h_{2}$ which form the first fan-ring have the same change trend. It can be observed that the low resonant frequency is the result of the combination of $h_{1}$ and $h_{2}$, the two jointly affect the low resonant frequency and bandwidth; 2) It shows that size of the largest sector-loop is correlated with the wavelength of low $\left(f_{1}\right.$ and $f_{2}$ ) resonant frequency, which can be used to figure out sidelength of the sector-loop.

With fan-shaped nested structure, the distance between each unit is reduced, which will lead to a coupling increase. Under the interaction of different nested loop and especially for the high frequency pass-band, mainly influenced by the internal sector in gand affected by inner and outer ring simultaneously, the relationship between the center frequency for the high frequency pass-band and the ring size is complex, while the adjustment for the low frequency pass-band is relatively easy. 


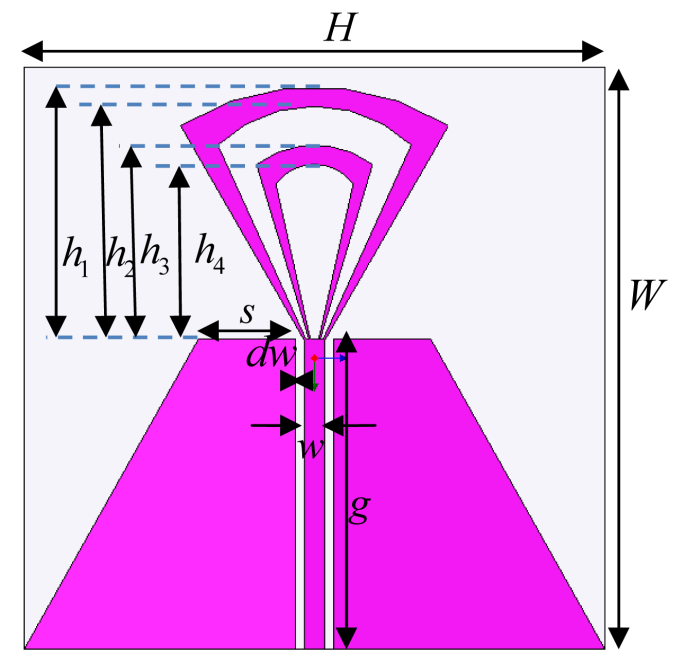

Figure 2. Antenna structure.

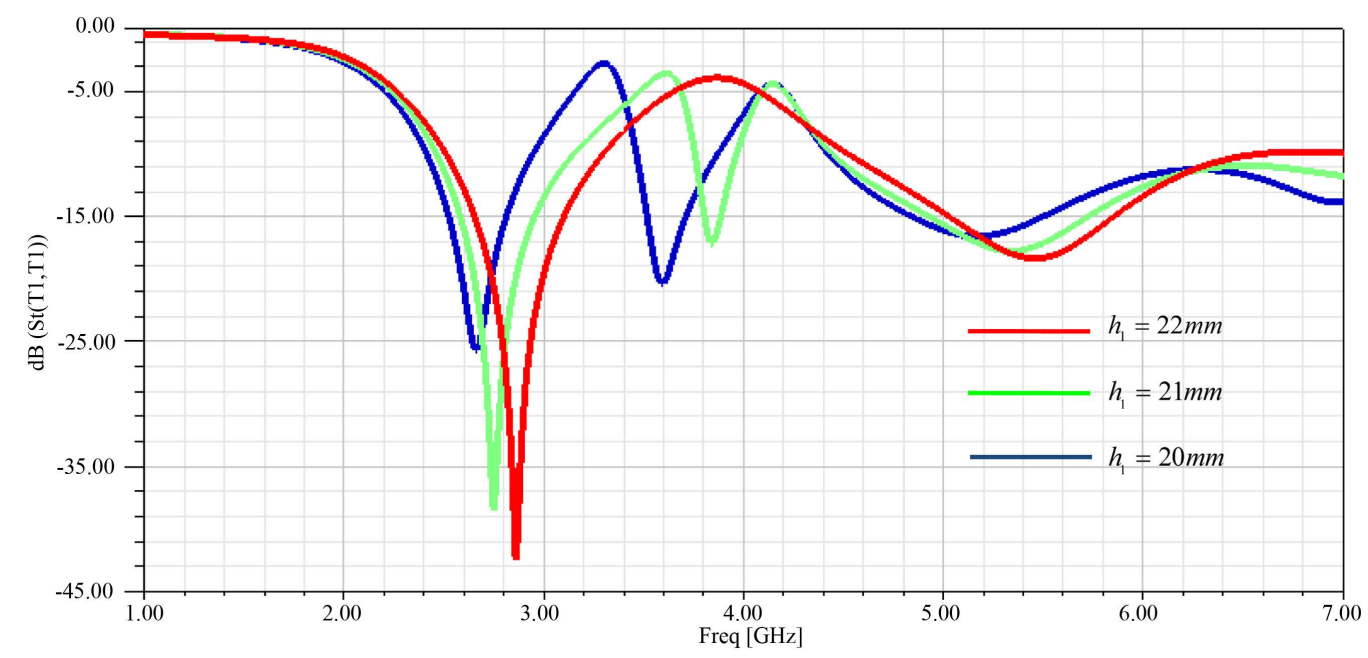

Figure 3. Parametric studies of effect of $h_{1}$ on the return loss of antenna.

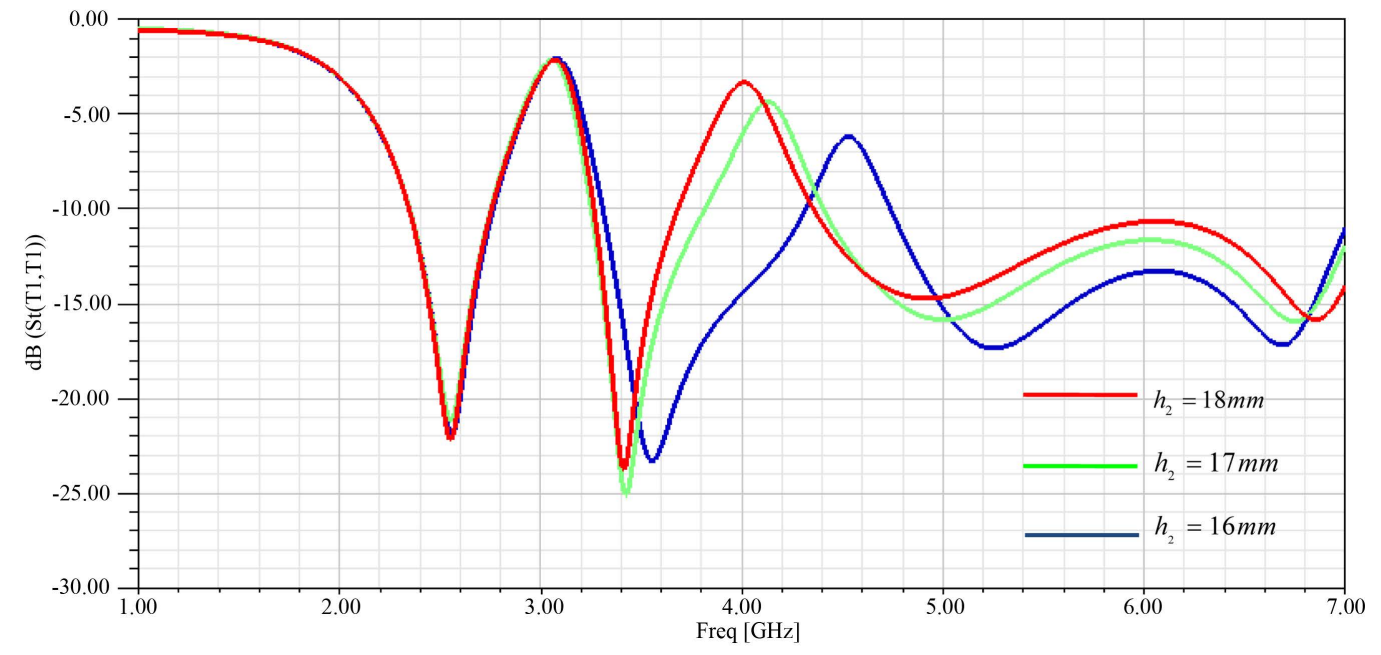

Figure 4. Parametric studies of effect of $h_{2}$ on the return loss of antenna. 


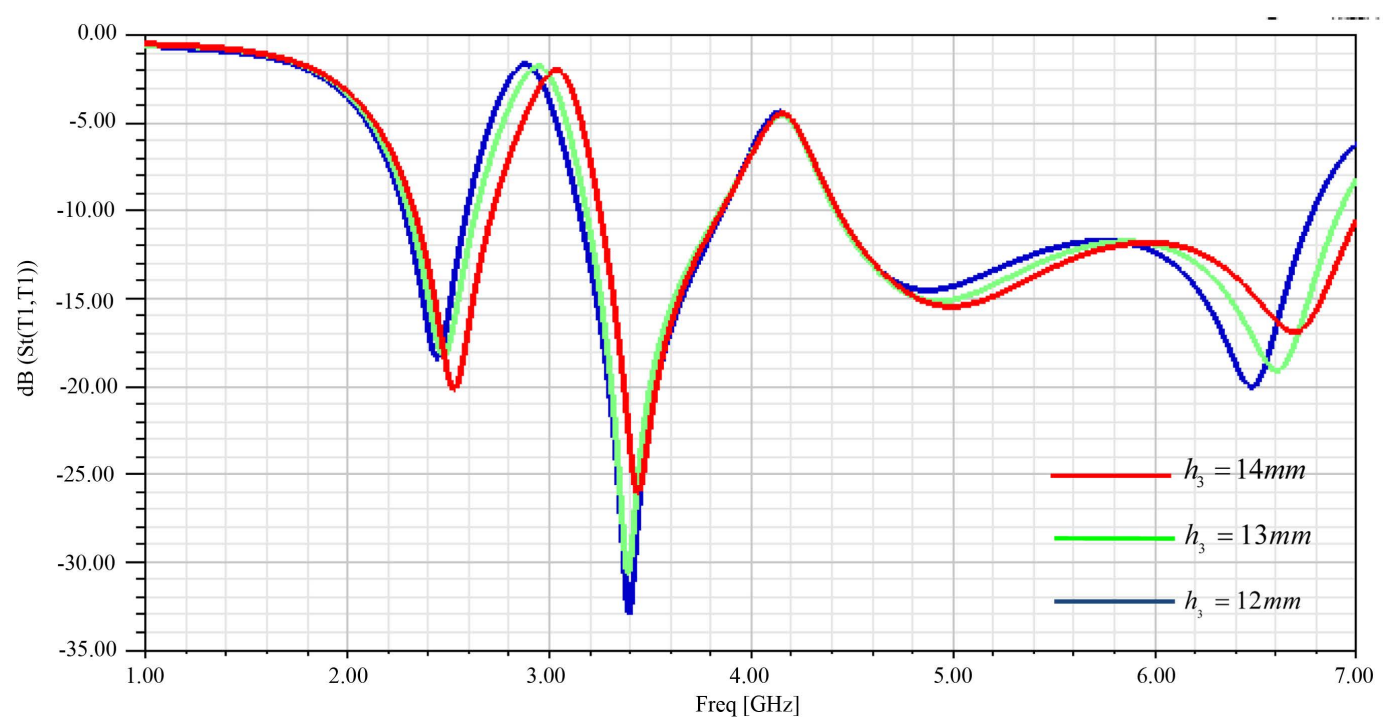

Figure 5. Parametric studies of effect of $h_{3}$ on the return loss of antenna.

Table 1. Summaryof the pass-bands with different heights.

\begin{tabular}{|c|c|c|c|c|c|c|c|}
\hline & & \multicolumn{2}{|l|}{$f_{1}$} & \multirow{2}{*}{ Center Freq (GHz) } & \multicolumn{3}{|c|}{$f_{3}$} \\
\hline & & Center Freq (GHz) & $\mathrm{S}_{11}(\mathrm{~dB})$ & & $\mathrm{S}_{11}(\mathrm{~dB})$ & Center Freq (GHz) & $\mathrm{S}_{11}(\mathrm{~dB})$ \\
\hline \multirow{3}{*}{$h_{1}$} & 22 & 2.61 & -23.4 & - & - & 5.41 & -17.3 \\
\hline & 21 & 2.74 & -39.0 & 3.92 & -16.8 & 5.44 & -17.9 \\
\hline & 20 & 2.83 & -41.3 & 3.58 & -21.3 & 5.49 & -18.7 \\
\hline \multirow{3}{*}{$h_{2}$} & 18 & 2.528 & -21.8 & 3.63 & -23.8 & 4.86 & -14.8 \\
\hline & 17 & 2.578 & -22.3 & 3.38 & -24.2 & 5.12 & -16.2 \\
\hline & 16 & 2.586 & -22.6 & 3.41 & -25.1 & 5.23 & -17.6 \\
\hline \multirow{3}{*}{$h_{3}$} & 14 & 2.48 & -20.6 & 3.42 & -27.3 & 5.01 & -13.2 \\
\hline & 13 & 2.473 & -18.8 & 3.40 & -33.1 & 4.98 & -14.3 \\
\hline & 12 & 2.46 & -18.6 & 3.43 & -32.8 & 4.96 & -14.9 \\
\hline
\end{tabular}

\subsection{The Fan Apex Angle}

Keep the nested sector remain the same, that is, to meet the conditions: $h_{1}=21 \mathrm{~mm}, h_{2}=17 \mathrm{~mm}, h_{3}=14 \mathrm{~mm}$, $h_{4}=12 \mathrm{~mm}, \theta_{2}=70^{\circ}, \theta_{3}=60^{\circ}, \theta_{4}=50^{\circ}$. Change the nested fan apex angle and observe the change of antenna pass-band. Because the nested sector number is large, and nested fan transverse size will change with angle adjustment, so this paper only makes a qualitative analysis on the angle change of the outermost layers $\left(\theta_{1}\right)$.

As $\theta_{1}$ grows, antenna pass-band bandwidth also increases significantly, the pass-band center frequency and reflection loss $\left(\mathrm{S}_{11}\right)$ have non-monotone variation, Figure 6 shows the antenna's $\mathrm{S}_{11}$. Apex angle size will affect the ultra-wideband antenna characteristics, and in order to achieve multiple frequency characteristics, the internal fan-shaped apex angle should not be too small, and outer fan apex angle should not be too big. Thus in this paper we choose $\theta_{1}=80^{\circ}$.

Through the above analysis, we can draw the following conclusions: the fan nested fractal antenna has the characteristic of multiple frequency, and the height and apex angle of the nested fan will influence the frequency, size of the pass-band, and the depth of resonance of the multi-frequency pass-band. According to the characteristics of the fan nested fractal antenna and the researches of the effects of different parameters on the band, we can design a multi-band antenna with three bands $(2.48 \mathrm{GHz}, 3.5 \mathrm{GHz}$ and $5.58 \mathrm{GHz}$ ) by adjusting values of the parameters. As the fan apex angle has a small contribution on frequency of the antenna, the antenna design is 
accomplished mainly by adjusting the fan height. Because the adjustment for the low frequency pass-band is relatively easier than that for the high frequency, so we should first determine the lowest center frequency as $2.48 \mathrm{GHz}$, and then set the second and the third band center frequency $(3.5 \mathrm{GHz}, 5.58 \mathrm{GHz}$ ) by adjusting the nested triangular height. If the first center frequency is $2.48 \mathrm{GHz}$, we can determine the outer triangle height according to formula (3). The experiential formula of antenna size can be established from simulation, which indicates the relation between fan side-length and the wavelength of corresponding resonant frequency, as is shown in the following: $l=0.14 \sim 0.15 \lambda$.

According to the above discussion and in order to realize the multi-bands, the structural parameters of this antenna can be set as: $h_{1}=21 \mathrm{~mm}, h_{2}=17 \mathrm{~mm}, h_{3}=14 \mathrm{~mm}, h_{4}=12 \mathrm{~mm}, \theta_{1}=80^{\circ}, \theta_{2}=70^{\circ}, \theta_{3}=60^{\circ}$, $\theta_{4}=50^{\circ}, H=36 \mathrm{~mm}, W=42 \mathrm{~mm}, s=8 \mathrm{~mm}, d w=0.9 \mathrm{~mm}, g=23 \mathrm{~mm}, w=1.3 \mathrm{~mm}$.

Figure 7 shows the antenna simulated results. It can be seen from the simulated results that the antenna has operating bands of $2.38-2.785 \mathrm{GHz}, 3.21-3.82 \mathrm{GHz}$ and $4.42-6.825 \mathrm{GHz}$ respectively, and the bandwidths can meet the requirement of WLAN/WiMAX communication. Figure 8 gives the simulated radiation patterns at 2.48, 3.5, and $5.58 \mathrm{GHz}$. The structure of the proposed antenna is symmetrical in E plane, therefore the pattern is also symmetric in E plane and is a figure of eight in E Plane. It can be seen that this antenna has the nearly omni-directional radiation characteristics, while the cross-polarization level rises with frequency increases owing to the horizontal components of the surface currents. In addition, consistency of the pattern exists for all frequencies in the operating band.

Figure 9 gives the antenna gain within WLAN/WiMAX frequencies, it can be seen from the diagram that in the low frequency the gain is around $2.1-3 \mathrm{~dB}$, and in high frequencies it is around $4-5 \mathrm{~dB}$, thus the optimal gain characteristic are suitable for mobile devices.

In Table 2, $H_{i}$ represents the height of antenna, $\lambda_{i}$ is the wavelength corresponding to the $i$ th resonant

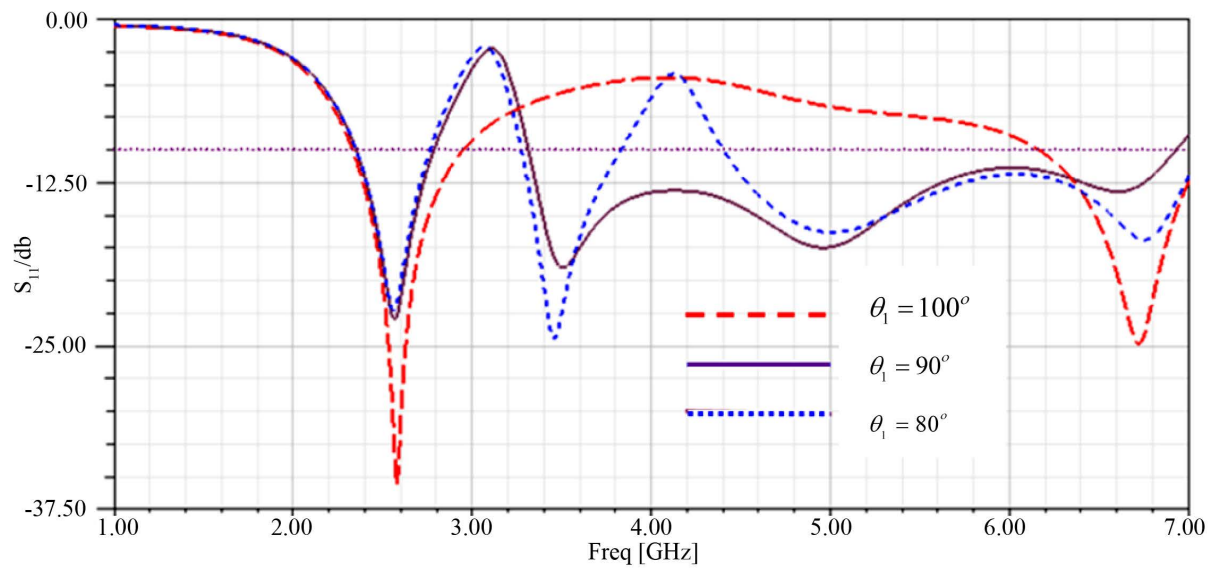

Figure 6. Parametric studies of effect of $\theta_{1}$ on the return loss of antenna.

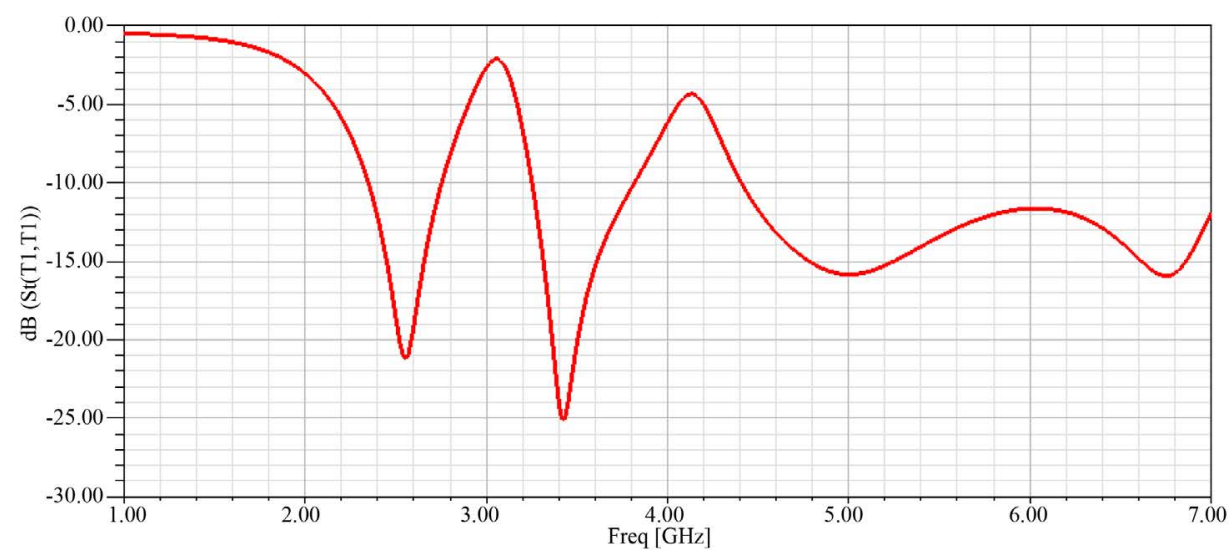

Figure 7. Simulated return loss of the proposed antenna. 

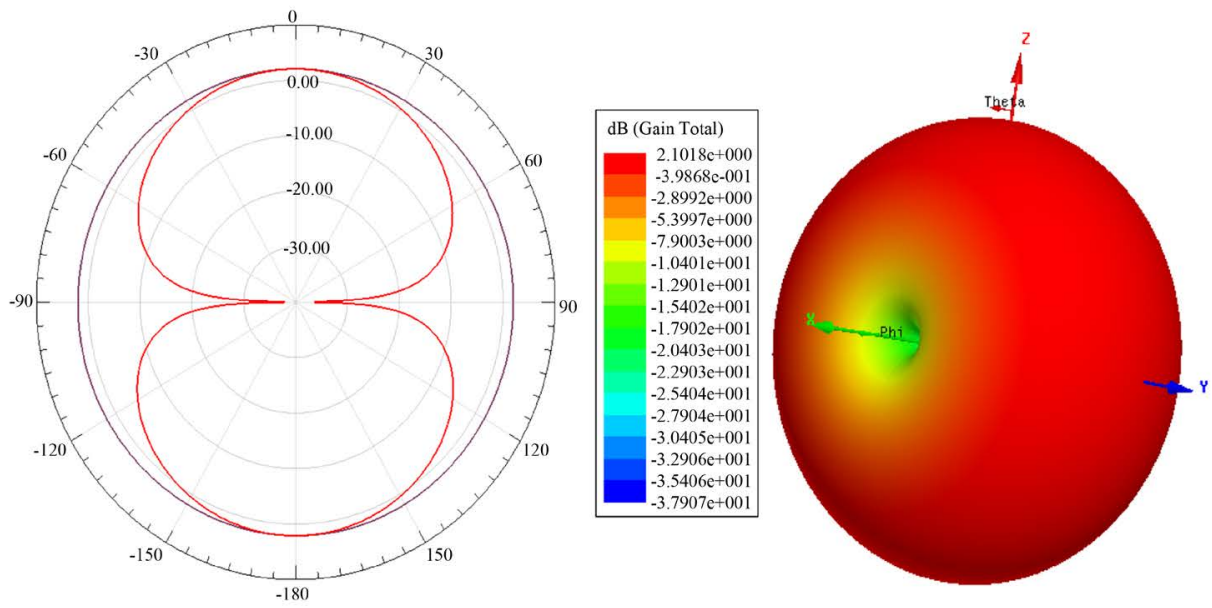

(a) $f_{1}=2.48 \mathrm{GHz}$
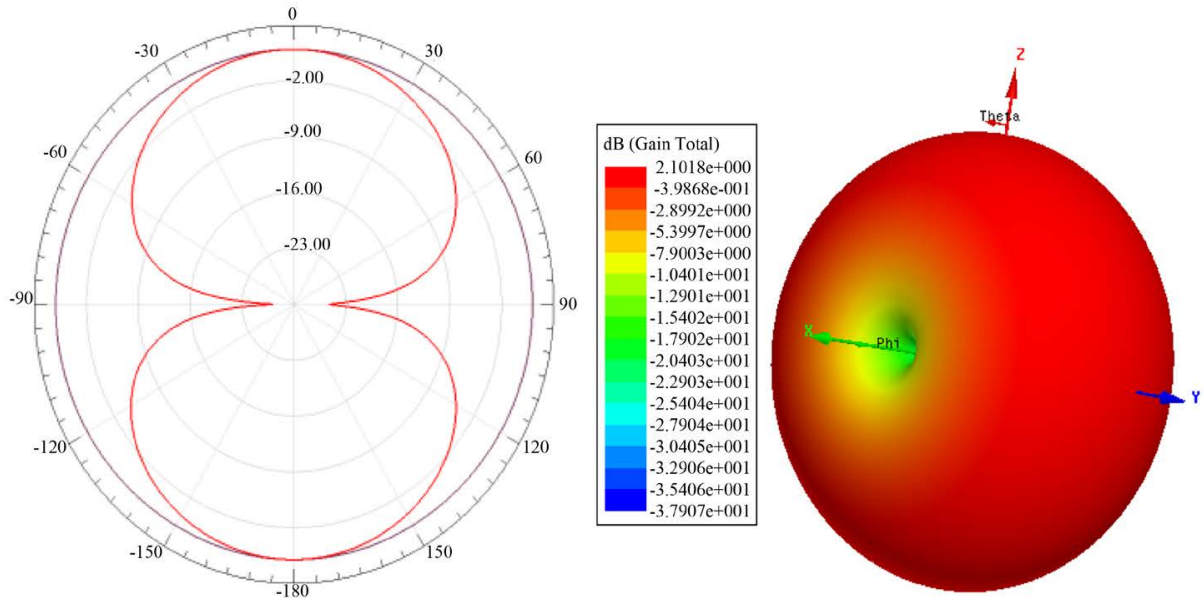

(b) $f_{2}=3.5 \mathrm{GHz}$
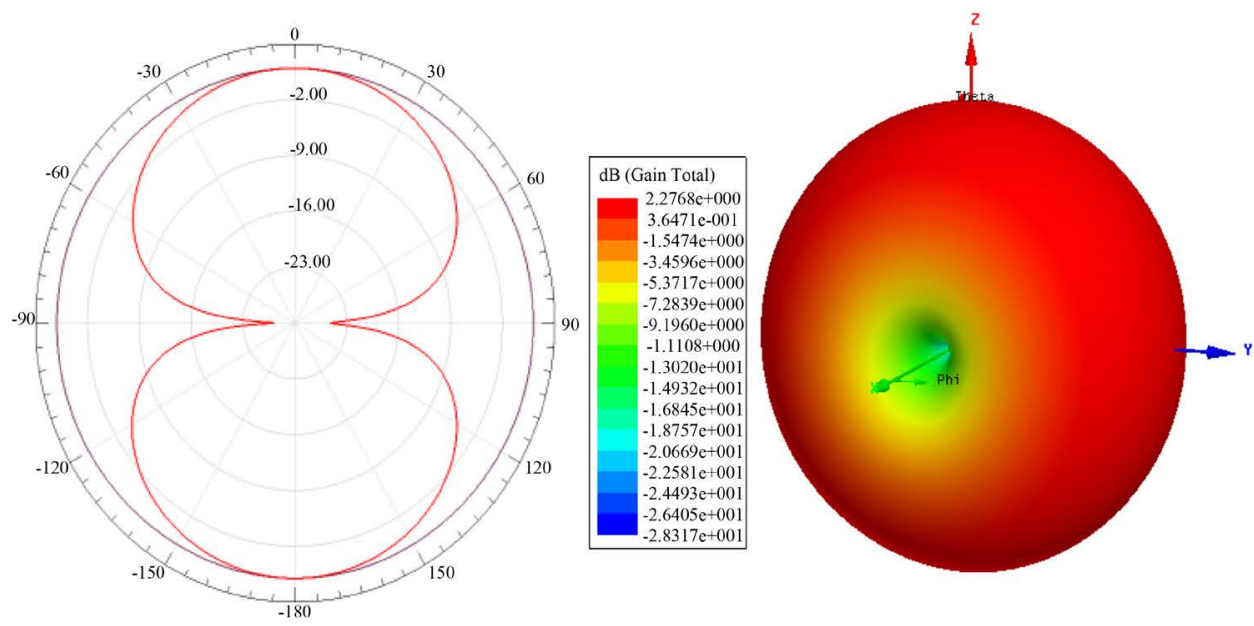

(c) $f_{3}=5.58 \mathrm{GHz}$

Figure 8 . The simulated results radiation patterns. 


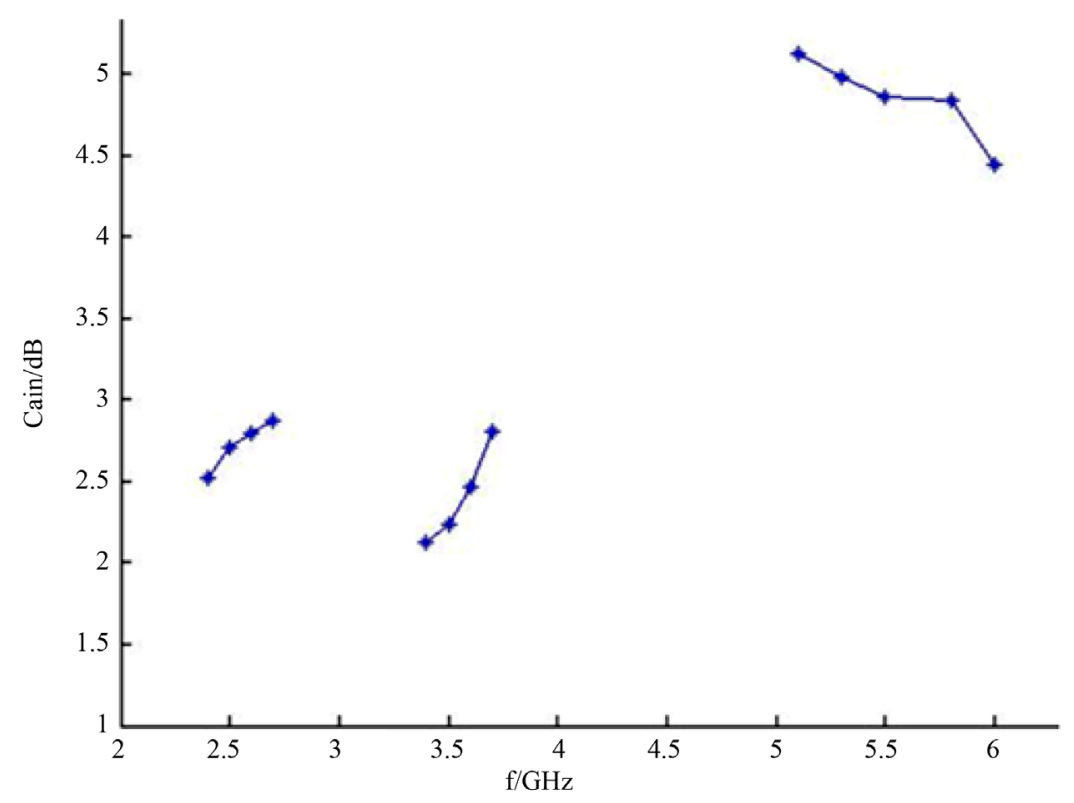

Figure 9. Simulated gains.

Table 2. Characterof the types of muti-frequency antennas.

\begin{tabular}{cccc}
\hline & & \multicolumn{3}{c}{$\kappa_{i}=H_{i} / \lambda_{i}$} \\
\cline { 2 - 4 } Antenna & Center Value of $\kappa_{i}$ & Range of $\kappa_{i}$ & Largest Deviation of $\kappa_{i}$ \\
\hline Proposed & 0.117 & $0.112-0.122$ & 4.3 \\
Diamond Monopole [14] & 0.350 & $0.234-0.465$ & 33.0 \\
Antenna [15] & 0.375 & $0.369-0.381$ & 10.6 \\
Antenna [16] & 0.263 & $0.242-0.283$ & 7.8 \\
\hline
\end{tabular}

frequency. The center value of $\kappa_{i}$ is the initial value of multi-frequency antenna size, and the smaller the value, the smaller of the antenna height. For the largest deviation of $\kappa_{i}$, the bigger the value, the worse the antenna frequency controllability. It is noted that the area of this antenna is only about $0.117 \lambda$, while those of [14]-[16] are $0.35 \lambda, 0.375 \lambda, 0.263 \lambda$ respectively. Therefore, among these designs, the proposed sector-nested fractal antenna has the smallest area and the best controllability, which principally comes from the use of the trapeziform ground plane and the sector-nested fractal structure.

\section{Conclusions}

A novel sector-nested fractal antenna has been introduced. By combining two techniques: the sector-nested fractal structure and the trapeziform ground plane, the monopole multiband antenna is designed to cover three bands at $2.44 \mathrm{GHz} / 3.5 \mathrm{GHz} / 5.2 \mathrm{GHz}-5.8 \mathrm{GHz}$, which are required by WLAN/WiMAX systems. In addition, the antenna has simple structure, thin profile, low cost and significant gain. Therefore it can be applied for the electronic protection systems, etc., and will be an attractive candidate for various WLAN/WiMAX applications.

This paper is too short to provide sufficient evidence about general properties of fractal antennas and to infer much about their relationship with the antenna fractal dimension and its performance indices. Nevertheless, our results indicate that a good analysis framework has been established for further study of miniature multi-band antennas for practical applications. For future work, we intend to investigate the reduction in size without diminishing the performance of antennas, and to seek its application in wireless systems.

\section{Acknowledgements}

This work was supported by the Natural Science Foundation of Guangdong Province, China (Grant No. 
2010B050900016), the fundamental research funds for the Central Universities (Grant No. 21613323) and the Funds for International Cooperation and Exchange of the National Natural Science Foundation of China (Grant No. 21614605).

\section{References}

[1] Pourahmadazar, J., Ghobadi, C., Nourinia, J. and Shirzad, H. (2012) Multi-Band Ring Fractal Antenna for Mobile Devices. IEEE Antennas and Wireless Propagation Letters, 9, 863-866.

[2] Best, S.R. (2012) On the Resonant Properties of the Koch Fractal and Other Wire Monopole Antennas. IEEE Antennas and Wireless Propagation Letters, 1, 74-76. http://dx.doi.org/10.1109/LAWP.2002.802550

[3] Sundram, A., Maddela, M. and Ramadoss, R. (2007) Koch-Fractal Folded-Slot Antenna Characteristics. IEEE Antennas and Wireless Propagation Letters, 6, 219-222. http://dx.doi.org/10.1109/LAWP.2007.895293

[4] Best, S.R. (2013) On The Performance Properties of the Koch Fractal and Other Bent Wire Monopoles. IEEE Transactions on Antennas and Propagation, 51, 1292-1300. http://dx.doi.org/10.1109/TAP.2003.812257

[5] Mirzapour, B. and Hassani, H.R. (2008) Size Reduction and Bandwidth Enhancement of Snowflake Fractal Antenna. IET Microwaves, Antennas \& Propagation, 2, 180-187. http://dx.doi.org/10.1049/iet-map:20070133

[6] Huang, C.Y. and Hsia, W.C. (2005) Planar Elliptical Antenna for Ultra-Wideband Communications. Electronics Letters, 41, 296-297. http://dx.doi.org/10.1049/el:20057244

[7] Chen, K.R., Sim, C.Y.D. and Row, J.S. (2013) A Compact Monopole Antenna for Super Wideband Applications. IEEE Antennas and Wireless Propagation Letters, 10, 488-491. http://dx.doi.org/10.1109/LAWP.2011.2157071

[8] Deng, C., Xie, Y.J. and Li, P. (2009) CPW-Fed Planar Printed Monopole Antenna with Impedance Bandwidth Enhanced. IEEE Antennas and Wireless Propagation Letters, 8, 1394-1397.

[9] Dong, Y., Hong, W., Liu, L., Zhang, Y. and Kuai, Z. (2009) Performance Analysis of a Printed Super-Wideband Antenna. Microwave and Optical Technology Letters, 51, 949-956. http://dx.doi.org/10.1002/mop.24222

[10] Baliarda, C.P., Romeu, J. and Cardama, A. (2000) The Koch Monopole: A Small Fractal Antenna. IEEE Transactions on Antennas and Propagation, 48, 1773-1781. http://dx.doi.org/10.1109/8.900236

[11] Karim, M.N.A., Rahim, M.K.A., Ayop, O.B., Abu, M. and Zubir, F. (2013) Log Periodic Fractal Koch Antenna for UHF Band Applications. Progress in Electromagnetic Research (PIER), 100, 201-218. http://dx.doi.org/10.2528/PIER09110512

[12] Best, S.R. (2012) The Effectiveness of Space-Filling Fractal Geometry in Lowering Resonant Frequency. IEEE Antennas and Wireless Propagation Letters, 1, 112-115. http://dx.doi.org/10.1109/LAWP.2002.806050

[13] Venoy, K.J., Abraham, J.K. and Varadan, V.K. (2003) Fractal Dimension and Frequency Response of Fractal Shaped Antennas. Proceedings of IEEE Antennas and Propagation Society International Symposium, 4, 222-225.

[14] Rumsey, V. (1966) Frequency Independent Antennas. Academic, New York.

[15] Zhong, S.S., Liang, X.L. and Wang, W. (2007) Compact Elliptical Monopole Antenna with Impedance Bandwidth in Excess of 21:1. IEEE Transactions on Antennas and Propagation, 55, 3080-3085.

[16] Ma, T.G. and Jeng, S.K. (2005) Planarminiature Tapered-Slot-Fed Annular Slotantennas for Ultra Wideband Radios. IEEE Transactions on Antennas and Propagation, 53, 1194-1202. http://dx.doi.org/10.1109/TAP.2004.842648 